\title{
Le barriere reattive permeabili per la bonifica delle acque sotterranee
}

\author{
Vincenzo Ricciardi ${ }^{1}$ \\ ${ }^{1}$ University of Salerno
}

\begin{abstract}
L'inquinamento, provocato da attività agricole e industriali, può causare la contaminazione delle matrici ambientali, costituendo un rischio significativo per l'approvvigionamento di acqua potabile e per gli ecosistemi. Pertanto, negli Stati Uniti e in Europa si sono sviluppate, nel corso degli anni, diverse tecniche di bonifica. In particolare, per il risanamento di acque sotterranee, un metodo in situ relativamente nuovo e potenzialmente importante è la barriera reattiva permeabile (BRP). Essa prevede la realizzazione di una barriera all'interno della zona satura del terreno con materiali reattivi per il trattamento di contaminanti organici e inorganici presenti in forma disciolta nell'acqua di falda. L'articolo illustra il funzionamento delle BRP attraverso l'esposizione di un caso studio, dove tale tecnologia ha permesso di ridurre le concentrazioni di uranio nelle acque sotterranee di un sito di stoccaggio.
\end{abstract}

\section{Le barriere reattive permeabili}

La contaminazione delle acque sotterranee e superficiali è diventata, in tutto il mondo, un problema che riguarda l'ambiente e la salute pubblica. Infatti, essa crea un rischio significativo per l'approvvigionamento di acqua potabile e influisce negativamente sugli ecosistemi, ad esempio causando l'eutrofizzazione. Nel corso degli anni, si sono sviluppate diverse tecniche per la bonifica di acque e suolo contaminati da sostanze pericolose. In particolare, le barriere reattive permeabili (permeable reactive barriers, PRBs) sono una tecnica di bonifica in situ relativamente nuova e potenzialmente importante. Tale tecnica consente la degradazione e la rimozione di contaminanti organici e inorganici presenti in forma disciolta nell'acqua di falda. È una tecnologia di bonifica passiva in quanto consiste nella realizzazione di una barriera all'interno della zona satura del terreno con materiali reattivi. Una volta realizzata l'installazione, la barriera non impedisce il flusso naturale delle acque ma sfrutta il gradiente della falda (figura 1). Quindi, la barriera reagisce con i contaminanti disciolti in acqua, degradandoli 
attraverso reazioni di ossidoriduzione con la formazione di composti meno "tossici". Tutto questo permette di ridurre le concentrazioni dei contaminanti a valori compatibili con i limiti stabiliti dalla normativa ambientale.

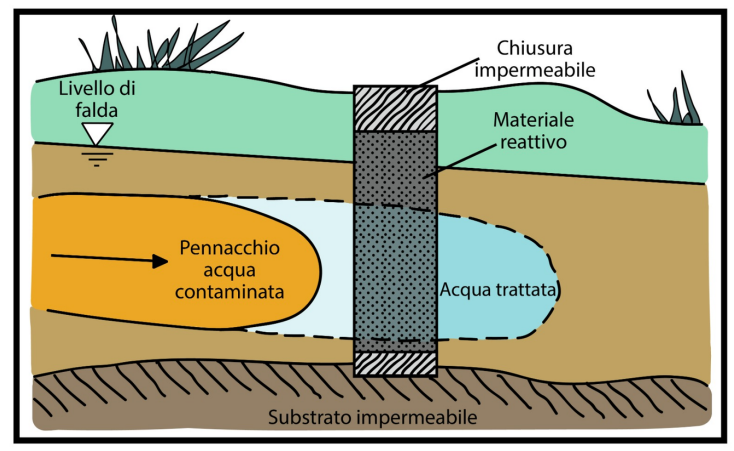

Figure 1: Schema di funzionamento barriera reattiva permeabile

Il materiale impiegato per la BRP deve essere reattivo verso i contaminanti da rimuovere. Inoltre, il materiale non deve decadere facilmente e non deve essere solubile in acqua. Per evitare l'aggiramento della barriera, è necessario che la permeabilità della zona reattiva della stessa sia maggiore o uguale a quella dell'acquifero. Tra il materiale reattivo e i contaminati si sviluppano reazioni di diversa natura, le quali possono formare degli intermedi di reazione ed è importante che questi non siano più tossici dei materiali di partenza. Diversi sono i materiali che possono costituire una barriera reattiva permeabile (leghe bimetalliche, zeoliti, carboni attivi, carbonati e idrossidi di calcio, ditionite di sodio ecc), ma ad oggi il più utilizzato risulta il ferro zero valente $\left(\mathrm{Fe}^{0}\right)$. Le barriere attive permeabili, a seconda del materiale utilizzato, si distinguono in biologiche, chimiche e adsorbenti $^{1}$.

Le BRP, essendo un sistema passivo, non necessitano di impianti fuori terra, rendendo l'area immediatamente fruibile. Esse consentono di degradare pennacchi di contaminazione con sorgenti distribuite o difficilmente localizzabili, grazie al fatto che possono essere realizzate in diverse configurazioni. Le più note sono quella funnel-and-gate e quella continua. Per quanto riguarda i costi associati alle barriere reattive permeabili, essi sono legati principalmente alla fase iniziale di scavo per l'inserimento della barriera e al monitoraggio per la verifica del trattamento (costi di gestione minimi). Tuttavia, le BRP sono efficaci solo per i composti disciolti e il sistema è fortemente dipendente dal flusso di falda. Un'altra problematica riguarda la longevità e la necessità di rigenerare i materiali reattivi. In tal senso, poichè le BRP sono una tecnologia relativamente nuova, la durata media 
delle barriere si attesta sui 15 anni.

\section{Riferimenti normativi}

II riferimento normativo, in ambito italiano, per la bonifica dei siti contaminati è il D.Lgs. 152/2006 - parte IV, titolo V. Esso definisce quelle che sono le concentrazioni soglia di contaminazione (Csc), cioè i livelli di contaminazione delle matrici ambientali al di sopra dei quali è necessaria la caratterizzazione del sito e l'analisi di rischio sito specifica, e le concentrazioni soglia di rischio ( $\mathrm{Csr}$ ) cioè i livelli di contaminazione delle matrici ambientali da determinare caso per caso con l'applicazione della procedura di analisi rischio sito specifica, il cui superamento richiede la messa in sicurezza e la bonifica. Nel caso delle barriere reattive permeabili importante risulta essere la tabella 2 presente nell'allegato 5 della parte IV, titolo $\mathrm{V}$, in cui vengono definite le concentrazioni soglia di contaminazione per le acque sotterranee. In ambito internazionale, una normativa a cui spesso si fa riferimento è quella dell'agenzia statunitense per la protezione dell'ambiente (USEPA). In essa, riguardo alle acque, si fa riferimento, per ogni contaminante, a un livello massimo ammissibile ( $\mathrm{MCL}$ ) da dover considerare. In merito all'uranio, contaminate presente nel caso studio, la normativa italiana non stabilisce un valore soglia da rispettare, limite presente, invece, nella normativa americana, la quale pone un limite per l'uranio pari a 0,03 $\mathrm{mg} / \mathrm{L}$.

\section{II successo delle BRP}

Pur essendo una tecnica di bonifica relativamente nuova, in tutto il mondo si contano più di $200 \mathrm{BRP}$ installate con successo. Queste installazioni, si trovano principalmente negli Stati Uniti dove la US Environmental Protection Agency (EPA) ha designato le BRP come tecnologia standard di bonifica per le acque sotterranee ${ }^{2}$. Restando in nord America, in Canada ci sono esempi di BRP per la rimozione di metalli pesanti ${ }^{3}$. In Europa va segnalata la prima BRP europea per la rimozione di nitrati da acque sotterranee ${ }^{4}$. Non mancano installazioni in parti del mondo dove sussistono condizioni "estreme" come la BRP per la rimozione di metalli pesanti e idrocarburi, presso la Casey Station, in Antartide ${ }^{5}$. La ricerca attiva riguardo le barriere reattive permeabili fa sì che si sperimentino sempre nuovi materiali ${ }^{6}$, nuovi metodi per rigenerare i materiali reattivi ${ }^{7}$, nuove configurazioni ${ }^{8}$ e talvolta accoppiamenti con altre tecniche per migliorare le efficienze di bonifica ${ }^{9}$. Infine, in alcuni casi, le barriere reattive permeabili vengono implementate per realizzare interventi di messa in sicurezza al fine di isolare il pennacchio della contaminazione ed impedirne la propagazione. 


\section{Caso studio: un nuovo modello di barriera}

Il caso studio illustra le fasi di progettazione e installazione di una BRP per la rimozione di uranio dalle acque sotterranee ${ }^{10}$. Una città, situata nel bacino minerario di Kryvyi Rig, a causa delle attività industriali associate alla lavorazione dell'uranio, ha fatto registrare un significativo inquinamento delle acque sotterranee. Per ovviare a questa problematica si è deciso di intervenire installando una BRP. II primo passo è stato la caratterizzazione del sito. In particolare, si tratta di un sito di stoccaggio di sterili provenienti dalla lavorazione di uranio, situato a circa $1,5 \mathrm{~km}$ a sud di Zhovty Vody nel canale Shcherbakovskaya, affluente del fiume Zhovta. Per molti anni il percolato di questa struttura si è infiltrato nel sottosuolo, contaminando le acque sotterranee sottostanti. La stratificazione del sottosuolo è composta da sottili strati superficiali di terre argillose, argille e sabbie (circa 3,5 $\mathrm{m}$ di spessore) che ricoprono uno strato di granito abbastanza permeabile (che raggiunge una profondità di $6 \mathrm{~m}$ ) nel quale è situata la falda. Le acque sotterranee nel granito fluiscono verso est dall'area della struttura di stoccaggio e scaricano verso il fiume Zhovta. I dati di monitoraggio vicino all'impianto, provenienti da una rete di 8 pozzi di monitoraggio, hanno indicato un pennacchio di contaminazione delle acque sotterranee ad alta concentrazione di nitrati e solfati. Inoltre, tale pennacchio segue la direzione del flusso delle acque sotterranee. II campionamento geochimico dettagliato delle acque ha rivelato la presenza di ioni metallici $\left(\mathrm{Na}^{+}, \mathrm{K}^{+}\right.$, $\left.\mathrm{Ni}^{2+}, \mathrm{Cu}^{2+}, \mathrm{Zn}^{2+}, \mathrm{Pb}^{2+}, \mathrm{Cd}^{2+}, \mathrm{Fe}_{\text {tot }}, \mathrm{Co}_{\text {tot }}, \mathrm{Mn}_{\text {tot }}\right)$, altri componenti chimici $\left(\mathrm{Mg}^{2+}, \mathrm{Ca}^{2+}, \mathrm{NH}_{4}{ }^{+}, \mathrm{HCO}_{3}{ }^{-}, \mathrm{Cl}^{-}, \mathrm{SO}_{4}{ }^{2-}, \mathrm{NO}_{3}{ }^{-}\right.$, solidi sospesi totali TDS) e uranio disciolto $\left(U_{\text {tot }}\right)$. Nello specifico, le concentrazioni di uranio $(0,42 \mathrm{mg} / \mathrm{L})$ superavano in maniera significativa i livelli massimi stabiliti dall'USEPA, che per l'uranio sono $0,03 \mathrm{mg} / \mathrm{L}$.

Per individuare i materiali e mezzi reagenti piu adatti per la BRP, sono stati effettuati una serie di esperimenti di laboratorio seguendo un approccio chimico e un approccio microbiologico. L'approccio chimico e stato perseguito attraverso batch tests (assenza di flusso) e test su colonna (flusso gravitazionale). I batch test hanno consentito di individuare i materiali reagenti, determinando l'efficienza di rimozione degli stessi in soluzioni di uranio contenenti agenti complessanti, ossia sostanze presenti nelle acque sotterranee che tendono a formare complessi con ioni $\mathrm{U}(\mathrm{VI})$. A tale scopo sono stati testati tre diversi tipi di ferro zero valente. II primo campione (ZVI1) era un ferro spugnoso (Fe $>99,5 \%$ ) con particelle di dimensioni < $0,16 \mathrm{~mm}$, il secondo campione (ZVI2) era un ferro (Fe $>90 \%)$ con dimensioni delle particelle $>1,0 \mathrm{~mm}$ e un terzo campione (ZVI3) di rottami di ferro con dimensioni delle particelle comprese tra $1,0 \mathrm{~mm}$ e 2,0 $\mathrm{mm}$. Con i test in colonna sono state testate tre miscele di materiali come riempimento per la BRP. In particolare, la prima miscela conteneva ferro zero valente in polvere (ZVI2) e sabbia (riempimento attivo inorganico), la seconda ferro zero valente in polvere $(\mathrm{ZVI} 2)$ con ghiaia, farina di ossa, segatura e attiva- 
tore (riempimento attivo organico-inorganico), e la terza ghiaia, farina di ossa, segatura e attivatore (riempimento attivo organico). Comunita batteriche anaerobiche sono state usate come attivatore per avviare i processi di bioriduzione dell'uranio.

Per valutare i diversi mezzi reattivi, sul sito da trattare si e installato una BRP con configurazione innovativa, formata da 21 cilindri suddivisi in tre gruppi da 7. Ogni gruppo di pozzi conteneva un diverso mezzo di trattamento. Infatti, il primo aveva come mezzo di riempimento attivo inorganico, il secondo un riempimento attivo organico-inorganico e il terzo un riempimento attivo organico. Tutti i cilindri avevano un diametro di 0,35 $\mathrm{m}$ e una profondita di $6 \mathrm{~m}$ tale da intercettare il flusso sotterraneo della falda. Inoltre, 8 pozzi di monitoraggio (3 a monte della BRP, 2 all'interno della BRP e altri 3 a valle della BRP) sono stati installati per campionare e verificare le efficienze di rimozione dell'uranio.

I risultati dei batch tests, hanno mostrato che il campione ZVI1 permetteva di avere la massima rimozione di uranio. Inoltre, si e dimostrato che la presenza di ligandi naturalmente presenti nelle acque sotterranee, che formano complessi con gli ioni $\mathrm{U}(\mathrm{VI})$, puo influenzare considerevolmente la reattivita dei materiali ferrosi, facendo diminuire l'efficienza di rimozione dell'uranio. Gli esperimenti in colonna, invece, hanno dimostrato una rimozione quasi completa di $\mathrm{U}(\mathrm{VI})$. Infine, i risultati ottenuti dai pozzi di monitoraggio installati nei pressi della barriera reattiva permeabile hanno indicato una notevole riduzione della contaminazione da uranio delle acque sotterranee. In particolare, i cilindri piu performanti sono stati quelli composti da materiale di riempimento inorganico e quelli con riempimento organico-inorganico.

\section{Conclusioni}

II caso studio ha riguardato le fasi di progettazione e installazione di una barriera reattiva permeabile (BRP), per la rimozione di uranio nelle acque sotterranee, nei pressi della citta di Zhovty Vody, in Ucraina. Le indagini condotte sul sito hanno indicato che l'uranio risultava essere presente in concentrazione superiore al limite massimo stabilito dall'USEPA $(0,03 \mathrm{mg} / \mathrm{L})$. Dopo due anni di monitoraggio, lo studio riporta che la concentrazione di uranio, dal valore iniziale di $0,42 \mathrm{mg} / \mathrm{L}$, e stata ridotta ad un valore compreso tra 0,07-0,10 mg/L. Quindi, la tecnica della BRP ha mostrato un'efficienza elevata. E' possibile incrementare l'efficienza di rimozione di questa tipologia di barriera, aggiungendo un maggior numero di file sfalsate di cilindri. Inoltre, il design innovativo della barriera, costituita da file di cilindri con materiali ferro-reattivi, ha consentito una notevole riduzione dei costi di installazione, rispetto alle barriere convenzionali. In definitiva, la barriera reattiva permeabile risulta essere una tecnica di bonifica economicamente e tecnicamente valida per il trattamento in situ 
di metalli e composti organici disciolti nelle acque sotterranee.

\section{References}

1.Striegel, J., Sanders, D. A. \& Veenstra, J. N. Treatment of Contaminated Groundwater Using Permeable Reactive Barriers. Environmental Geosciences 8, 258-265 (2001).

2.Permeable Reactive Barrier: Technology Update. The Interstate Technology \& Regulatory Council (2011).

3.McGregor, R., Benner, S., Ludwig, R., Blowes, D. \& Ptacek, C. Sulfate Reduction Permeable Reactive Barriers to Treat Acidity Cadmium, Copper, Nickel, and Zinc. in Handbook of Groundwater Remediation using Permeable Reactive Barriers 495-522 (Elsevier, 2003). doi:10.1016/b978012513563-4/50021-9.

4.Gibert, O., Assal, A., Devlin, H., Elliot, T. \& Kalin, R. M. Performance of a field-scale biological permeable reactive barrier for in-situ remediation of nitrate-contaminated groundwater. Science of The Total Environment 659, 211-220 (2019).

5.Statham, T. M., Stark, S. C., Snape, I., Stevens, G. W. \& Mumford, K. A. A permeable reactive barrier (PRB) media sequence for the remediation of heavy metal and hydrocarbon contaminated water: A field assessment at Casey Station Antarctica. Chemosphere 147, 368-375 (2016).

6.Rojas, C. M. M., Velasquez, M. F. R., Tavolaro, A., Molinari, A. \& Fallico, C. Use of Vegetable Fibers for PRB to Remove Heavy Metals from Contaminated Aquifers-Comparisons among Cabuya Fibers Broom Fibers and ZVI. International Journal of Environmental Research and Public Health 14, 684 (2017).

7.Falciglia, P. P. et al.. Field technical applicability and cost analysis for microwave based regenerating permeable reactive barriers (MW-PRBs) operating in Cs-contaminated groundwater treatment. Journal of Environmental Management 260, 110064 (2020).

8.Flury, B., Eggenberger, U. \& Mäder, U. First results of operating and monitoring an innovative design of a permeable reactive barrier for the remediation of chromate contaminated groundwater. Applied Geochemistry 24, 687-696 (2009).

9.Yoon, J. H., Kim, Y. N., Shin, D. C., Kim, K. R. \& Kim, K. H. Management of Animal Carcass Disposal Sites Using a Biochar Permeable Reactive Barrier and Fast Growth Tree (Populus euramericana): A Field Study in Korea. Sustainability 9, 457 (2017). 
10.Kornilovych, B. et al.. Uranium Removal from Groundwater by Permeable Reactive Barrier with Zero-Valent Iron and Organic Carbon Mixtures: Laboratory and Field Studies. Metals 8, 408 (2018). 